\title{
Body size at birth modifies the effect of fat mass and obesity associated (FTO) rs9939609 polymorphism on adiposity in adolescents: the Healthy Lifestyle in Europe by Nutrition in Adolescence (HELENA) study
}

Idoia Labayen ${ }^{1,2,3 *}$, Jonatan R. Ruiz ${ }^{3,4}$, Francisco B. Ortega ${ }^{3,5}$, Frédéric Gottrand ${ }^{6,7}$, Inge Huybrechts ${ }^{8}$, Jean Dallongeville ${ }^{9}$, Kurt Widhalm ${ }^{10}$, Marika Ferrari ${ }^{11}$, Annete Buyken ${ }^{12}$, Mathilde Kersting ${ }^{12}$, George Moschonis $^{13}$, Dominique Turck ${ }^{6,7}$, Sonia Gómez ${ }^{14}$, Michael Sjostrom ${ }^{4}$, Aline Meirhaeghe ${ }^{9}$ and Luis A. Moreno ${ }^{2,15}$

${ }^{1}$ Department of Nutrition and Food Science, University of the Basque Country, Paseo de la Universidad, 7 , 01006 Vitoria-Gasteiz, Spain

${ }^{2}$ GENUD (Growth, Exercise, Nutrition and Development) Research Group, Zaragoza, Spain

${ }^{3}$ Unit for Preventive Nutrition, Department of Biosciences and Nutrition at NOVUM, Karolinska Institutet, SE-17177

Huddinge, Sweden

${ }^{4}$ Department of Physical Activity and Sport, School of Physical Activity and Sport Sciences, University of Granada, Granada, Spain ${ }^{5}$ Department of Medical Physiology, School of Medicine, University of Granada, 18014 Granada, Spain

${ }^{6}$ INSERM U995, IFR 114, Faculty of Medicine, University of Lille 2, Lille, France

${ }^{7}$ Department of Pediatrics, Jeanne de Flandre Children's University Hospital, Lille, France

${ }^{8}$ Department of Public Health, School of Medicine, Ghent University, Ghent, Belgium

${ }^{9}$ INSERM U744, Institut Pasteur de Lille, UDSL, Lille F-59000, France

${ }^{10}$ Division of Nutrition and Metabolism, Department of Pediatrics, Medical University of Vienna, Vienna, Austria

${ }^{11}$ INRAN (National Research Institute on Food and Nutrition), Rome, Italy

${ }^{12}$ Research Institute of Child Nutrition Dortmund, Rheinische Friedrich-Wilhelms-Universität Bonn, Dortmund, Germany

${ }^{13}$ Department of Nutrition and Dietetics, Harakopio University, Athens, Greece

${ }^{14}$ Immunonutrition Research Group, Department of Metabolism and Nutrition, Spanish Council for Scientific Research (CSIC), Madrid, Spain

${ }^{15}$ University School of Health Sciences, University of Zaragoza, Zaragoza, Spain

(Submitted 14 April 2011 - Final revision received 15 July 2011 - Accepted 15 July 2011 - First published online 15 September 2011)

Abstract

The present study was intended to examine whether ponderal index (PI) at birth modifies the effect of the fat mass and obesity associated (FTO) rs9939609 polymorphism on adiposity in European adolescents. A total of 628 adolescents aged $14 \cdot 4$ (SE 1.3 ) years (56.8\% female) were recruited. PI was calculated from parental reports of birth weight and length $\left(\mathrm{kg} / \mathrm{m}^{3}\right)$, and the BMI $\left(\mathrm{kg} / \mathrm{m}^{2}\right)$, body fat percentage and fat mass index (FMI, $\mathrm{kg} / \mathrm{m}^{2}$ ) were calculated. The rs 9939609 polymorphism was genotyped and physical activity assessed by accelerometry. Sex, duration of pregnancy, pubertal status, centre and physical activity were used as confounders in all the analyses. The minor A allele of the FTO rs9939609 was significantly associated with higher BMI, body fat percentage and FMI (all $P<0 \cdot 05$ ) but not with PI. Significant interactions between PI and the rs9939609 polymorphism in terms of body fat percentage $(P=0 \cdot 002)$ and FMI $(P=0 \cdot 017)$ were detected. However, this polymorphism was only significantly associated with higher BMI, body fat percentage and FMI (all $P<0 \cdot 05$ ) in adolescents in the lower PI tertile. Indeed, both body fat percentage and FMI were higher in those adolescents in the lower PI tertile carrying the A allele of the FTO rs9939609 polymorphism than in those with the TT genotype (25.0 (SE 0.8) v. 22.1 (sE 1.0) \%, adjusted $P=0 \cdot 030$ and 5.6 (sE $0 \cdot 3$ ) v. $4.6(\mathrm{SE} 0.4) \mathrm{kg} / \mathrm{m}^{2}, P=0.031$, respectively). Our findings suggest that those adolescents born with lower PI could be more vulnerable to the influence of the A risk allele of the FTO polymorphism on total adiposity content.

Key words: Fat mass and obesity associated gene: Adiposity: Intra-uterine growth: Adolescents

Abbreviations: FMI, fat mass index; FTO, fat mass and obesity associated; HELENA, Healthy Lifestyle in Europe by Nutrition in Adolescence; PI, ponderal index. *Corresponding author: Dr Idoia Labayen, fax + 34 945130756, email idoia.labayen@ehu.es 
The development of adiposity, and its complications, is determined by lifestyle factors, genetic mechanisms and their interactions $^{(1)}$. The genetic factors include SNP in genes that encode proteins involved in biological processes which influence, among others, body composition. Although one of the main genetic susceptibility factors for obesity discovered to date is the so-called fat mass and obesity associated (FTO) gene $^{(2)}$, its role in energy homeostasis remains to be elucidated $^{(3)}$. Recently, Larder et al. ${ }^{(4)}$ reviewed the findings from human and murine genetic studies which have explored the potential role of the FTO protein, a member of the Fe(II)and 2-oxoglutarate-dependent oxygenase superfamily, in the regulation of energy homeostasis and metabolism. These authors concluded that many unanswered questions remain in relation to the physiological role of FTO. We have shown previously that the minor A allele of the FTO rs9939609 polymorphism is significantly associated with higher serum leptin concentrations in adolescents, independent of potential confounders including adiposity ${ }^{(3)}$. Indeed, our findings suggested that leptin could be a possible intermediary contributing to the association between the FTO rs9939609 polymorphism and adiposity. Common polymorphisms located in the first intron of the FTO gene are associated with a predisposition to obesity in populations of different ethnic background or age ${ }^{(5)}$.

Several studies ${ }^{(6-8)}$ have suggested that the deleterious effect of the FTO polymorphism can be attenuated by physical activity. Indeed, we have recently reported that physical activity may attenuate the effect of the FTO rs9939609 polymorphism on adiposity in a cohort of European adolescents ${ }^{(9)}$. Thus, we found that adolescents meeting the daily physical activity recommendations ( $60 \mathrm{~min}$ or more of moderate to vigorous physical activity per d) can overcome the effect of the FTO rs9939609 polymorphism on obesity-related traits.

Epidemiological findings and results from experimental studies have highlighted an association between small body size at birth and many metabolic disorders ${ }^{(10-12)}$ and unhealthy body composition later in life ${ }^{(13,14)}$. Indeed, it has been suggested that insufficient nutrient supply during fetal life could lead to permanent changes in the structure and function of certain organs and tissues ${ }^{(11)}$. A small body size at birth may programme smaller proportions of lean tissue mass later in life, thus leading such individuals to be more vulnerable to the additional effects of lifestyle when nutrient supply is abundant.

Genetic susceptibility is important in the determination of both birth weight and adult metabolic disorders ${ }^{(15)}$. Ponderal index (PI) at birth, a measure of how heavy the newborn is for a given length, is an estimate of newborn nutritional status. However, it is also considered to be an indirect indicator of the intra-uterine environment, which could influence gene expression and lead to phenotypes associated with disease. Likewise, genes related to adiposity and CVD may have different effects on individuals with different body size at birth ${ }^{(16)}$

The aims of the present study were (i) to examine whether body size at birth modifies the effect of the FTO rs9939609 polymorphism on adiposity in European adolescents and (ii) to assess whether physical activity influences this association.

\section{Materials and methods}

\section{Study design}

The Healthy Lifestyle in Europe by Nutrition in Adolescence (HELENA) study was designed to examine the interactions between personal, environmental and lifestyle influences on the risk factors for future CVD. Data were collected during 2006 and 2007 in ten European cities, namely Athens, Heraklion, Dortmund, Ghent, Lille, Pecs, Rome, Stockholm, Vienna and Zaragoza. A detailed description of the HELENA study sampling and recruitment approaches, standardisation and harmonisation processes, data collection, analysis strategies and quality-control activities has been published elsewhere $^{(17)}$.

\section{Subjects}

All adolescents meeting the general HELENA inclusion criteria (not participating simultaneously in another clinical trial, and being free of any acute infection lasting less than 1 week before inclusion), with valid data for age, sex and BMI, were taken as the final HELENA sample (3546 adolescents aged $12.5-17.5$ years). In order to investigate clinical biochemistry assays and genetic analyses, one-third of the participants were randomly selected for blood collection, resulting in a total of 1144 subjects. The present study comprises 909 adolescents (501 females) with valid data regarding FTO rs9939609 polymorphism and body weight and length at birth. We also identified 628 participants with valid data regarding physical activity (Fig. 1). The final sample did not differ in terms of the main characteristics (i.e. neonatal characteristics such as body weight, length and PI at birth, age or BMI) from the original sample (all $P>0 \cdot 1$ ).

Written informed consent to participate was obtained from both parents and adolescents. The study was performed

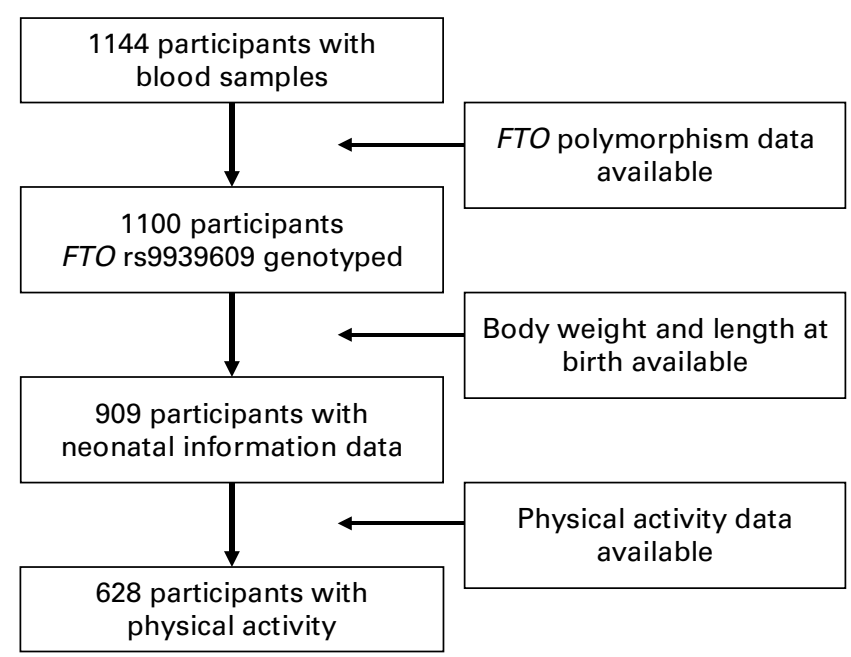

Fig. 1. Flow diagram of participants. FTO, fat mass and obesity associated gene. 
following the ethical guidelines of the Declaration of Helsinki 1961 (revision of Edinburgh 2000), Good Clinical Practice and current legislation regarding clinical research in humans in each of the participating countries. The protocol was approved by the Human Research Review Committee of the Universities and centres involved.

\section{Neonatal data}

Body weight and length at birth, and duration of gestation, were collected from parental records using a questionnaire. Parents were asked to recall this information from the health booklets of their son/daughter ${ }^{(18)}$. Duration of gestation was reported in three categories: less than 37 weeks, between 37 and 40 weeks and more than 40 weeks. This questionnaire was sent to the parents together with the study information letter and consent form, and collected at school on the first day of the examinations. If information from the parental questionnaire was lacking, the local investigators were advised to send the questionnaire to the parents again to obtain the required information. PI was computed as birth weight $(\mathrm{kg})$ divided by birth length $(\mathrm{m})$ cubed.

\section{Physical examination}

Harmonisation and standardisation of the anthropometric measurements used to assess body composition in the HELENA study were strictly controlled and have been described previously ${ }^{(19)}$. Body weight and height, and subscapular and tricipital skinfold thicknesses, were measured in triplicate. BMI was calculated as body weight (kg) divided by the square of the height $(\mathrm{m})$. The body fat percentage was calculated using Slaughter's equation:

Body fat $(\%)=1 \cdot 21($ tricipital + subscapular $)-0 \cdot 008($ tricipital

$$
+ \text { subscapular }^{2}-3 \cdot 4
$$

which showed the best agreement with total body fat percentage measured by dual-energy X-ray absorptiometry in the adolescent population $^{(20)}$, and thereafter the fat mass index (FMI) was calculated by dividing fat mass by height squared (m). This index adjusts for current body size in a similar way to that used for BMI. Identification of sexual maturation (stages $\mathrm{I}-\mathrm{V}$ ) was assessed by a medical doctor according to Tanner \& Whitehouse ${ }^{(21)}$.

\section{Assessment of physical activity}

Physical activity was assessed using a uni-axial accelerometer (Actigraph $^{\text {TM }}$ GT1M, Pensacola, FL, USA) attached to the lower back. Adolescents were instructed to wear the accelerometer for $7 \mathrm{~d}$, during all waking hours, and to remove it only during water-based activities. At least $3 \mathrm{~d}$ of recording with a minimum of $8 \mathrm{~h}$ registration/d was set as an inclusion criterion. The time sampling interval (epoch) was set at $15 \mathrm{~s}^{(22)}$.

\section{Genotyping}

The FTO rs9939609 genotyping was performed using an Illumina system equipped with GoldenGate technology (Illumina, Inc., San Diego, CA, USA). The genotyping success rate was $100 \%$. Overall, $2 \%$ of the sample was double-genotyped and the concordance rate was $99.9 \%$. The genotype distribution of the study sample respected the Hardy-Weinberg equilibrium $(P=0 \cdot 15)$.

\section{Statistical analysis}

The increase in weight, length and PI at birth variables per risk allele were determined using an additive genetic model after controlling for sex, duration of pregnancy and centre (entered as dummy variable). Trend tests were performed by adding genotype categories in the regression analysis as ordinal variables instead of categorical variables (i.e. $0=\mathrm{TT}, 1=\mathrm{TA}$, $2=\mathrm{AA}$ ).

The differences in adiposity estimates at adolescence (BMI, body fat percentage and FMI) between the three FTO rs9939609 genotypes were analysed using an additive model in two separate regression models. Thus, model 1 used sex, pubertal status and centre (entered as dummy variable) as potential confounders (fixed effects), whereas model 2 used sex, pubertal status, centre (entered as dummy variable) and physical activity as potential confounders (fixed effects). To test for the existence of an interaction between the FTO rs9939609 polymorphism and PI on body fat estimates, we used the same model as earlier but added a cross-product term $F T O \times$ PI into the model. In those cases where an interaction was detected (i.e. $P<0.05$ for $F T O \times$ PI), the analyses were performed stratifying by sex-specific tertiles of PI as follows: $<24 \cdot 6,24 \cdot 6-27 \cdot 1$ and $>27 \cdot 1 \mathrm{~kg} / \mathrm{m}^{3}$ for the low, middle and upper tertile, respectively, in girls, and $<24 \cdot 4,24 \cdot 4-27 \cdot 3$ and $>27 \cdot 3 \mathrm{~kg} / \mathrm{m}^{3}$ for the low, middle and upper tertile, respectively, in boys. The genotype distribution among PI tertiles, centres and pregnancy duration periods was compared using $\chi^{2}$ tests. The analyses were repeated stratifying by sex-specific birth weight tertiles instead of sex-specific PI.

Finally, the estimated means of body content (body fat percentage and FMI) by FTO rs9939609 genotype group (dominant model, TT $v$. A allele carriers) were calculated by performing an ANCOVA, adjusting for duration of pregnancy, sex, pubertal status and centre (entered as random variable) in model 1 and after additional adjustment for physical activity in model 2.

Analyses were performed using the Statistical Package for Social Sciences (SPSS, v. 17.0 for WINDOWS; SPSS Inc., Chicago, IL, USA) and the level of significance was set to 0.05.

\section{Results}

The neonatal and clinical characteristics of adolescents are shown in Table 1 . The frequency of the minor allele was $0 \cdot 40$. The distribution of the genotype frequencies of the FTO rs9939609 polymorphism did not differ significantly between the centres $(P>0 \cdot 05)$. 
Table 1. Descriptive characteristics of the Healthy Lifestyle in Europe by Nutrition in Adolescence study sample

(Number of subjects, percentages, mean values and standard deviations)

\begin{tabular}{|c|c|c|c|c|}
\hline & $n$ & $\%$ & Mean & SD \\
\hline \multicolumn{5}{|l|}{ Neonatal data } \\
\hline Birth weight $(\mathrm{kg})$ & 909 & & 3.33 & 0.56 \\
\hline Birth length (cm) & 909 & & $50 \cdot 4$ & $3 \cdot 1$ \\
\hline $\mathrm{Pl}$ at birth $\left(\mathrm{kg} / \mathrm{m}^{3}\right)$ & 909 & & $26 \cdot 2$ & $6 \cdot 6$ \\
\hline \multicolumn{5}{|l|}{ Duration of pregnancy } \\
\hline$<37$ weeks & 46 & $5 \cdot 1$ & & \\
\hline $37-40$ weeks & 574 & 63.1 & & \\
\hline$>40$ weeks & 289 & 31.8 & & \\
\hline \multicolumn{5}{|l|}{ Clinical characteristics } \\
\hline Females & 501 & $55 \cdot 1$ & & \\
\hline Males & 408 & 43.2 & & \\
\hline \multicolumn{5}{|l|}{ Pubertal status } \\
\hline Tanner stage 1 or 2 & 94 & $10 \cdot 4$ & & \\
\hline Tanner stage 3 or 4 & 556 & $61 \cdot 2$ & & \\
\hline Tanner stage 5 & 259 & 28.5 & & \\
\hline Age (years) & 909 & & 14.6 & 1.4 \\
\hline $\mathrm{BMI}\left(\mathrm{kg} / \mathrm{m}^{2}\right)$ & 909 & & $21 \cdot 2$ & $3 \cdot 7$ \\
\hline Body fat (\%) & 872 & & $23 \cdot 7$ & 9.5 \\
\hline FMI $\left(\mathrm{kg} / \mathrm{m}^{2}\right)$ & 872 & & $5 \cdot 28$ & 2.98 \\
\hline Physical activity (counts/min) & 628 & & 434 & 149 \\
\hline
\end{tabular}

$\mathrm{PI}$, ponderal index; FMI, fat mass index.

\section{Associations of the fat mass and obesity associated} rs9939609 polymorphism with neonatal characteristics and adiposity

The minor A allele of the FTO rs9939609 polymorphism was not significantly associated with birth weight, birth length or PI (Table 2).

As far as clinical parameters at adolescence were concerned, we observed that the minor A allele of the FTO rs9939609 polymorphism was significantly associated with higher BMI, body fat percentage and FMI after adjusting for sex, pubertal status and centre $(P<0 \cdot 005$, Table 2$)$.

Interactions between ponderal index and the fat mass and obesity associated rs9939609 polymorphism

Significant interaction effects were found between the FTO rs9939609 polymorphism and PI in its association with body fat percentage (adjusted $P=0.002$ ) and FMI (adjusted $P=0.017)$. After verifying that the distribution of the FTO rs9939609 polymorphism was similar among the PI tertiles, we stratified the sample according to sex-specific PI tertiles.

The A minor allele of the FTO rs9939609 polymorphism was only found to be significantly associated with increased BMI, body fat percentage and FMI regardless of sex, centre, duration of pregnancy and pubertal status in adolescents in the lower PI tertile (all adjusted $P<0 \cdot 01$, Table 3). Furthermore, these relationships remained statistically significant after additional adjustment for physical activity (all adjusted $P<0.05$, Table 3). Indeed, both body fat percentage and FMI were higher in those adolescents carrying the A allele of the FTO rs9939609 polymorphism than in those with the TT genotype, although only in the lower PI tertile (25.0 (SE 0.8) $v$. 22.0 (SE 0.9$) \%$, sex, centre, duration of pregnancy and pubertal status adjusted $P=0.008$ and 5.6 (SE 0.3$) v .4 .6$
(sE 0.4$) \mathrm{kg} / \mathrm{m}^{2}$, adjusted $P=0.008$, respectively, in model 1 , and $25 \cdot 0(\operatorname{SE} 0 \cdot 8) v \cdot 22 \cdot 1$ (SE $1 \cdot 0) \%$, sex, centre, duration of pregnancy, pubertal status and physical activity adjusted $P=0.030$ and $5.6($ SE 0.3$)$ v. $4.6($ SE 0.4$) \mathrm{kg} / \mathrm{m}^{2}$, adjusted $P=0.031$, respectively, in model 2; Fig. 2).

The results did not change when other cut-off points (i.e. sex-specific quartiles) were used instead of PI tertiles to classify the adolescents' PI (data not shown). When sex-specific birth-weight tertiles were used instead of PI tertiles, the association of the A allele of the rs9939609 polymorphism with either FMI $(P=0.084, P=0.203$ and $P=0.735$ for the lower, middle and upper tertiles, respectively) or body fat percentage ( $P=0.074, P=0.091$ and $P=0.747$ for the lower, middle and upper tertiles, respectively) was attenuated.

\section{Discussion}

Our findings suggest that body size at birth influences the association between the minor A allele of the FTO rs9939609 polymorphism and body fat content in European adolescents. Thus, the A allele was found to be significantly associated with higher adiposity content, but only in adolescents born within the lower sex-specific PI tertile irrespective of duration of pregnancy, sex, pubertal status and physical activity. As far as we are aware, this is the first study to examine the possible programming effect of intra-uterine environment on the association between the FTO gene and adiposity.

The fetal origin hypothesis proposes that the nutrient and hormonal milieu of the fetus alters gene expression, thus resulting in developmental adaptations that lead to permanent changes in physiology and metabolism which, in turn, can predispose to chronic diseases later in life $\mathrm{e}^{(11)}$. Our results suggest that individuals born with a lower PI $\left(<24.4 \mathrm{~kg} / \mathrm{m}^{3}\right.$ in boys and $<24.6 \mathrm{~kg} / \mathrm{m}^{3}$ in girls), a surrogate of adverse intra-uterine environment, are more susceptible to the deleterious effect of the A risk allele of the FTO rs9939609 polymorphism. These findings should, however, be taken with caution and need to be replicated in bigger samples, other ethnicities and in subjects of different age groups. However,

Table 2. Associations of the fat mass and obesity associated (FTO) rs9939609 polymorphism with neonatal anthropometric variables and adolescents' body fat content estimates

(Number of subjects, unstandardised coefficients and standard errors)

\begin{tabular}{lrrrr}
\hline & \multicolumn{4}{c}{ FTO rs9939609 (exposure) } \\
\cline { 2 - 5 } Outcomes & $n$ & \multicolumn{1}{c}{$\beta$} & \multicolumn{1}{c}{$\mathrm{SE}$} & $P_{\text {add }}$ \\
\hline Neonatal variables & & & & \\
$\quad$ Birth weight $(\mathrm{kg})$ & 909 & -0.004 & 0.032 & 0.905 \\
$\quad$ Birth length $(\mathrm{cm})$ & 909 & 0.016 & 0.184 & 0.930 \\
PI $\left(\mathrm{kg} / \mathrm{m}^{3}\right)$ & 909 & 0.340 & 0.418 & 0.417 \\
Anthropometric variablest & & & & \\
BMI $\left(\mathrm{kg} / \mathrm{m}^{2}\right)$ & 909 & 0.531 & 0.189 & 0.004 \\
Body fat $(\%)$ & 872 & 1.506 & 0.505 & 0.003 \\
FMI $\left(\mathrm{kg} / \mathrm{m}^{2}\right)$ & 872 & 0.510 & 0.161 & 0.002 \\
\hline
\end{tabular}

$\beta$, Unstandardised coefficient; $P_{\text {add }}$, additive model (the exposure, FTO polymorphism was categorised as an ordinal variable as follows: TT genotype $=0$; TA genotype $=1$ and AA genotype $=2$ ); PI, ponderal index; FMI, fat mass index.

* Adjusted for sex, duration of pregnancy and centre.

$\dagger$ Adjusted for sex, pubertal status and centre. 
we believe that the results provide further insights regarding the potential modulating effect of intra-uterine environment on the influence of certain CVD risk factors or adiposityassociated polymorphisms. Moreover, our findings underline the importance of careful follow-up of children born with small body size at birth to detect the development of metabolic abnormalities such as excess adiposity.

In agreement with previous studies, no significant associations between the FTO polymorphism and body weight, length and PI at birth were observed ${ }^{(2,23)}$. These findings suggest that the association between the FTO gene and fat mass in humans may develop in the early postnatal period $^{(24)}$, maintain its effect during later childhood and facilitate further BMI increases during adulthood ${ }^{(23)}$ by either influencing the 'input' of the energy balance ${ }^{(25)}$ or controlling energy expenditure ${ }^{(26)}$. Moreover, a previous report suggested that the FTO gene product is a good candidate for an epigenetic mark modifier and that the A allele may affect other adiposity-related genes promoter methylation depending on a particular fetal environment ${ }^{(27)}$. However, Bassols et al. ${ }^{(28)}$ showed that placental FTO expression was associated with increased fetal weight and length, and Cauchi et al. ${ }^{(29)}$ found that newborns homozygous for the FTO rs1421085 C allele had a higher PI at birth.

The percentage of adolescents born preterm was similar to that in other populations (approximately 5\%). Unfortunately, our data did not allow us to examine the influence of the FTO polymorphism on duration of pregnancy since gestational age data were recorded as a categorical variable.

There is compelling evidence that human obesity is a multifactorial disorder where both genes ${ }^{(30,31)}$ and lifestyle factors, including diet, physical activity or intra-uterine environment, are important contributors ${ }^{(1,16,32)}$. We have recently reported that physical activity may attenuate the deleterious effect of the FTO rs9939609 polymorphism on adiposity in the same cohort of adolescents ${ }^{(9)}$. However, in the present study, the association between the rs9939609 polymorphism and adiposity persisted in adolescents born in the lower PI tertile, even when the analysis was further controlled for objectively assessed physical activity level.

PI is a body proportionality index that provides information regarding a newborn's nutritional status and adiposity ${ }^{(33)}$. Recent reports suggest that this index should be considered in routine growth monitoring of newborns ${ }^{(34)}$. PI was categorised according to sex-specific tertiles in order to maintain a relatively large numbers of participants by tertile. Nevertheless, we repeated the analysis by using other cut-off points to define low PI, such as sex-specific quartiles; the results were not substantially different.

Several study limitations need to be addressed. First of all, neonatal variables were not obtained directly from hospital records. Likewise, although the analysis was restricted to 909 (628 with physical activity data) out of 1144 eligible adolescents, the study sample did not differ significantly from the sample of those excluded from the study, nor from the sample as a whole. Finally, because of the cross-sectional design of the study, we cannot establish at what age the interaction effect between the FTO gene and PI on adiposity could 

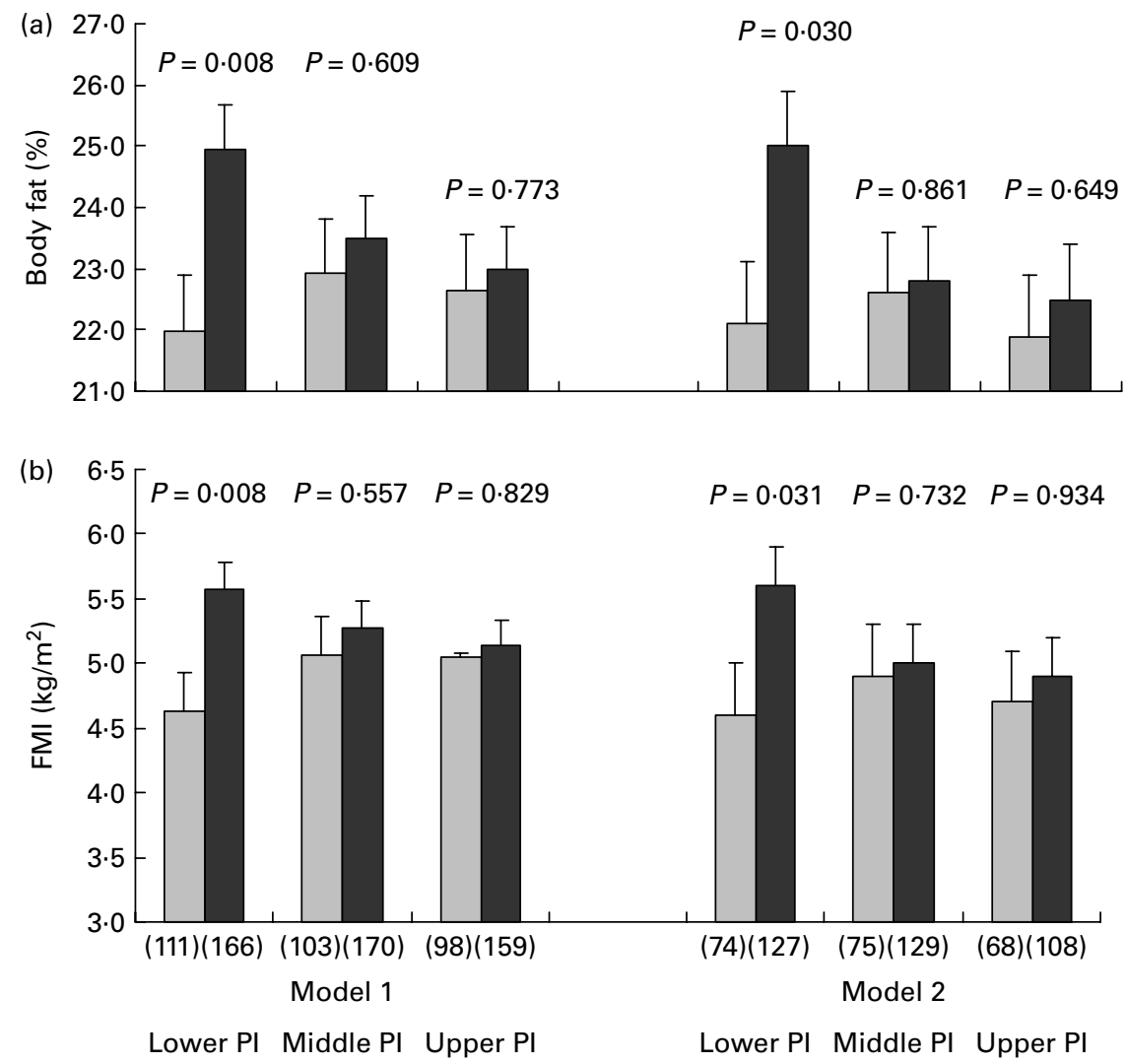

Fig. 2. Interaction effect between the fat mass and obesity associated (FTO) variant (rs9939609) and ponderal index (PI) tertiles (lower, middle and upper) on (a) body fat percentage and (b) fat mass index (FMI) in European adolescents. Values are means with their standard errors adjusted for duration of pregnancy, sex, age, pubertal status and centre (model 1), and additionally adjusted for physical activity (model 2, ANCOVA). Sample size in parentheses. $P$ for rs9939609 $\times$ PI tertiles on body fat percentage $=0.002$, and $P$ for rs $9939609 \times \mathrm{PI}$ tertiles on $\mathrm{FMI}=0.017$. $\square, \mathrm{TT} ; \mathrm{\square}, \mathrm{A}$ carriers.

start. The strength of the present study lay in the valid measurement of the phenotypes, including the objectively measured physical activity. The sample guaranteed a large geographical spread throughout Europe, and all measurements were obtained following standardised procedures at all the different centres.

In conclusion, our findings suggest that those adolescents born with a lower PI could be more vulnerable to the deleterious influence of the A risk allele of the FTO polymorphism on total adiposity content.

\section{Acknowledgements}

The authors' responsibilities were as follows: I. L. and J. R. R. wrote the manuscript; I. L. performed the statistical analysis; I. L., J. R. R., F. B. O., F. G., I. H., J. D., K. W., M. F., A. B., M. K., G. M., D. T., S. G., M. S., A. M. and L. A. M. contributed to the interpretation and discussion of the results. I. L. had full access to all the data in the study and takes responsibility for the integrity of the data and the accuracy of the data analysis. All authors contributed to the interpretation and discussion of the results, and critically revised the drafted manuscript. None of the authors had any personal or financial conflict of interest. The HELENA study was carried out with the financial support of the European Community Sixth RTD Framework
Programme (Contract FOODCT-2005-007034). This work was also partially supported by the Spanish Ministry of Science and Innovation (RYC-2010-05957), the Spanish Ministry of Education (EX-2008-0641), the Spanish Ministry of Health: Maternal, Child Health and Development Network (no. RD08/0072), the University of the Basque Country and the Swedish Heart-Lung Foundation (20090635). The writing group takes sole responsibility for the content of this article. The content of this paper reflects only the authors' views, and the European Community is not liable for any use that may be made of the information contained therein.

\section{References}

1. Loos RJ \& Rankinen T (2005) Gene-diet interactions on body weight changes. J Am Diet Assoc 105, Suppl. 1, 29-34.

2. Frayling TM, Timpson NJ, Weedon MN, et al. (2007) A common variant in the FTO gene is associated with body mass index and predisposes to childhood and adult obesity. Science 11, 889-894.

3. Labayen I, Ruiz JR, Ortega FB, et al. (2011) Association between the FTO rs9939609 polymorphism and leptin in European adolescents: a possible link with energy balance control. The HELENA study. Int J Obes (Lond) 35, 66-71.

4. Larder R, Cheung MK, Tung YC, et al. (2011) Where to go with FTO? Trends Endocrinol Metab 22, 53-59. 
5. Dina C, Meyre D, Gallina S, et al. (2007) Variation in FTO contributes to childhood obesity and severe adult obesity. Nat Genet 39, 724-726.

6. Vimaleswaran KS, Li S, Zhao JH, et al. (2009) Physical activity attenuates the body mass index-increasing influence of genetic variation in the FTO gene. Am J Clin Nutr 90, 425-428.

7. Andreasen $\mathrm{CH}$, Stender-Petersen KL, Mogensen MS, et al. (2008) Low physical activity accentuates the effect of the FTO rs 9939609 polymorphism on body fat accumulation. Diabetes 57, 95-101.

8. Rampersaud E, Mitchell BD, Pollin TI, et al. (2008) Physical activity and the association of common FTO gene variants with body mass index and obesity. Arch Intern Med 168, $1791-1797$.

9. Ruiz JR, Labayen I, Ortega FB, et al. (2010) Attenuation of the effect of the FTO rs9939609 polymorphism on total and central body fat by physical activity in adolescents: the HELENA study. Arch Pediatr Adolesc Med 164, 328-333.

10. Labayen I, Ortega FB, Sjostrom M, et al. (2009) Early life origins of low-grade inflammation and atherosclerosis risk in children and adolescents. J Pediatr 155, 673-677.

11. Barker DJ (2004) The developmental origins of adult disease. J Am Coll Nutr 23, Suppl. 6, 588-595.

12. Labayen I, Ruiz JR, Vicente-Rodriguez G, et al. (2009) Early life programming of abdominal adiposity in adolescents: the HELENA Study. Diabetes Care 32, 2120-2122.

13. Labayen I, Moreno LA, Blay MG, et al. (2006) Early programming of body composition and fat distribution in adolescents. J Nutr 136, 147-152.

14. Labayen I, Moreno LA, Ruiz JR, et al. (2008) Small birth weight and later body composition and fat distribution in adolescents: the AVENA Study. Obesity (Silver Spring) 16, 1680-1686.

15. Meirhaeghe A, Boreham CA, Murray LJ, et al. (2007) A possible role for the PPARG Pro12Ala polymorphism in preterm birth. Diabetes 56, 494-498.

16. Labayen I, Moreno LA, Marti A, et al. (2007) Effect of the Ala12 allele in the PPARgamma-2 gene on the relationship between birth weight and body composition in adolescents: the AVENA study. Pediatr Res 62, 615-619.

17. Moreno LA, De Henauw S, Gonzalez-Gross M, et al. (2008) Design and implementation of the Healthy Lifestyle in Europe by Nutrition in Adolescence Cross-Sectional Study. Int J Obes (Lond) 32, Suppl. 5, 4-11.

18. Iliescu C, Beghin L, Maes L, et al. (2008) Socioeconomic questionnaire and clinical assessment in the HELENA Cross-Sectional Study: methodology. Int J Obes (Lond) 32 , Suppl. 5, 19-25.

19. Nagy E, Vicente-Rodriguez G, Manios Y, et al. (2008) Harmonization process and reliability assessment of anthropometric measurements in a multicenter study in adolescents. Int $J$ Obes (Lond) 32, Suppl. 5, 58-65.
20. Rodriguez G, Moreno LA, Blay MG, et al. (2005) Body fat measurement in adolescents: comparison of skinfold thickness equations with dual-energy X-ray absorptiometry. Eur J Clin Nutr 59, 1158-1166.

21. Tanner JM \& Whitehouse RH (1976) Clinical longitudinal standards for height, weight, height velocity, weight velocity, and stages of puberty. Arch Dis Child 51, 170-179.

22. Ward DS, Evenson KR, Vaughn A, et al. (2005) Accelerometer use in physical activity: best practices and research recommendations. Med Sci Sports Exerc 37, Suppl. 11, $582-588$.

23. Jess T, Zimmermann E, Kring SI, et al. (2008) Impact on weight dynamics and general growth of the common FTO rs9939609: a longitudinal Danish Cohort study. Int J Obes (Lond) 32, 1388-1394.

24. Lopez-Bermejo A, Petry CJ, Diaz M, et al. (2008) The association between the FTO gene and fat mass in humans develops by the postnatal age of two weeks. J Clin Endocrinol Metab 93, 1501-1505.

25. Cecil JE, Tavendale R, Watt P, et al. (2009) An obesityassociated FTO gene variant and increased energy intake in children. $N$ Engl J Med 359, 2558-2566.

26. Fischer J, Koch L, Emmerling C, et al. (2009) Inactivation of the FTO gene protects from obesity. Nature 16, 894-898.

27. Gemma C, Sookoian S, Alvarinas J, et al. (2009) Maternal pregestational BMI is associated with methylation of the PPARGC1A promoter in newborns. Obesity (Silver Spring) 17, 1032-1039.

28. Bassols J, Prats-Puig A, Vazquez-Ruiz M, et al. (2010) Placental FTO expression relates to fetal growth. Int J Obes (Lond) 34, 1365-1370.

29. Cauchi S, Stutzmann F, Cavalcanti-Proenca C, et al. (2009) Combined effects of MC4R and FTO common genetic variants on obesity in European general populations. $J \mathrm{Mol}$ Med 87, 537-546.

30. Willer CJ, Speliotes EK, Loos RJ, et al. (2009) Six new loci associated with body mass index highlight a neuronal influence on body weight regulation. Nat Genet 41, 25-34.

31. Thorleifsson G, Walters GB, Gudbjartsson DF, et al. (2009) Genome-wide association yields new sequence variants at seven loci that associate with measures of obesity. Nat Genet 41, 18-24.

32. Marti A, Martinez-Gonzalez MA \& Martinez JA (2008) Interaction between genes and lifestyle factors on obesity. Proc Nutr SOC 67, 1-8.

33. Moyer-Mileur LJ, Slater H, Thomson JA, et al. (2009) Newborn adiposity measured by plethysmography is not predicted by late gestation two-dimensional ultrasound measures of fetal growth. J Nutr 139, 1772-1778.

34. Olsen IE, Lawson ML, Meinzen-Derr J, et al. (2009) Use of a body proportionality index for growth assessment of preterm infants. J Pediatr 154, 486-491. 